\title{
Quantum threshold reflection of He-atom beams from rough surfaces
}

\author{
G. Rojas-Lorenzo $\odot$ and J. Rubayo-Soneira* \\ Instituto Superior de Tecnologías y Ciencias Aplicadas, Universidad de La Habana, Avenida Salvador Allende No. 1110, \\ Quinta de Los Molinos, La Habana 10400, Cuba \\ S. Miret-Artés ${ }^{\dagger}$ \\ Instituto de Física Fundamental, Consejo Superior de Investigaciones Científicas, Serrano 123, 28006 Madrid, Spain \\ E. Pollak $\oplus^{*}$ \\ Chemical and Biological Physics Department, Weizmann Institute of Science, 76100 Rehovot, Israel
}

(Received 13 September 2019; revised manuscript received 20 December 2019; accepted 31 January 2020; published 24 February 2020)

\begin{abstract}
Quantum reflection of thermal He atoms from various surfaces (glass slide, GaAs wafer, flat, and structured $\mathrm{Cr}$ ) at grazing conditions is studied within the elastic close-coupling formalism. Comparison with the experimental results of Zhao et al. [Phys. Rev. Lett. 105, 133203 (2010)] is quite reasonable but the conclusions of the present theoretical analysis are different from those discussed in the experimental work. The universal linear behavior observed in the dependence of the reflection probability on the incident wave-vector component perpendicular to the surface is only valid at small values of the component whereas, at larger values, deviation from the linearity is evident, approaching a quadratic dependence at higher values. The surface roughness seems to play no important role in this scattering. Moreover, the claim that one observes a transition from quantum to classical reflection seems to be imprecise.
\end{abstract}

DOI: 10.1103/PhysRevA.101.022506

\section{INTRODUCTION}

Matter wave diffraction and interferometry are very interesting topics because, unlike optical effects observed by photons, these studies lead to a better knowledge of the interaction between particles and surfaces. This interaction is usually divided into two regions, short- and long-range distances. The short distances are dominated by the Pauli and Coulomb repulsion between electrons of the incoming particle and the surface and the long distances are governed mainly by the van der Waals-Casimir attraction. We talk about classical reflection when particles reach the inner region (turning points) corresponding to the repulsive part of the interaction potential. On the other hand, when the reflection comes from the long-range attractive part, one talks about quantum threshold reflection to be distinguished from above barrier quantum reflection. Lennard-Jones and Devonshire [1] first recognized this behavior in atom-surface scattering and Kohn [2] showed later on that quantum reflection leads to a zero sticking probability at threshold. He pointed out that this reflection is a quantum interference process between the incoming and reflected waves. Senn [3] showed that, for general one-dimensional forces which vanish as the coordinate goes to $\pm \infty$, the reflection probability goes to unity at threshold

\footnotetext{
*german@instec.cu,jrs@instec.cu

${ }^{\dagger}$ s.miret@iff.csic.es

¥eli.pollak@weizmann.ac.il
}

energies except when the potential supports a zero-energy resonance state. The reflection coefficient decreases from unity according to $|R| \sim 1-2 k b \sim \exp (-2 k b)$, where $k$ is the incident wave vector and $b$ is a characteristic length which depends on the specifics of the particle surface interaction. This universal behavior is a direct result of boundary conditions and continuity of the wave function and its derivative. When considering scattering from surfaces which occurs in three-dimensional space, $k$ should be replaced by its component perpendicular to the surface.

This quantum threshold reflection phenomenon has been observed for the scattering of ultracold metastable He atoms on silicon [4] and for rare-gas atoms and small clusters on gratings and surfaces [5-8] and on a periodic array of half planes [9]. In this last case, it appears there is a transition from quantum reflection to the regime where edge diffraction from half planes dominates. At threshold conditions, where the incident energy is very small, the maximum of the scattered wave function is located far away from the grating or surface due to the very long de Broglie wavelength of the incident particle. This led mistakenly to the idea that quantum reflection takes place far away from the grating or surface, at distances of typically tens or hundreds of nanometers. Quantum threshold reflection was claimed to be governed by the long-range attractive van der Waals-Casimir potential tail, which falls off faster than $r^{-2}[10,11]$. It was claimed that the fact that the very weakly bound $\mathrm{He}_{2}$ molecule which is reflected without dissociating is further proof that only the long-range weak forces are at play and these are too weak 
to dissociate even such a fragile bond [8]. This would not be the case if the dimer reaches the classical turning point of the interaction potential.

In the semiclassical framework, and within the $k$ linear dependence regime, the semiclassical description of the scattering dynamics breaks down. Far away from the grating or surface, the long-range attractive potential exhibits a region in which the local de Broglie wavelength is not slowly varying, invalidating a semiclassical description. This occurs in the so-called badlands region of the interaction potential. Quantum reflection was thus associated with this badlands region through a function called "quantality" the absolute value of which displays large deviations from unity in a confined region of the potential, implying that in this region quantum effects are important [11,12]. In a series of papers, we have recently demonstrated theoretically and numerically [13-15] that quantum threshold reflection is determined by the whole range of the interaction potential. We have observed that the short-range region is also critical for obtaining theoretical reflection probabilities and diffraction patterns which are in fairly good agreement with the experimental results of Refs. [5-8]. These calculations were carried out by using the elastic close-coupling (CC) formalism [16], which is numerically exact when convergence is reached. To distinguish between quantum and classical reflection in this type of theoretical calculations, complex absorbing boundary conditions which prevent the classical reflection from occurring have been employed [17,18]. In Ref. [14] we have also shown that the badlands region of the interaction potential is irrelevant in quantum threshold reflection since the wavelength of the scattering particles at threshold is much longer than the rather small spatial extent of this region.

In the work presented in this paper, we have extended our previous studies to analyze and discuss previously reported coherent reflection of He-atom beams from rough surfaces at grazing incidence [6]. When the component of the incident wave vector of the incoming atom perpendicular to the surface is very small, experimental and theoretical reflection probabilities seem to be only dependent on this normal component and approach unity when it vanishes, confirming the corresponding universal behavior. Deviations from linearity are found only at larger values of this normal component. Moreover, we do not find a transition from quantum to classical reflection when increasing the normal component of the wave vector, as previously reported [6]. Finally, the surface roughness at threshold conditions and grazing angles appears to play no important role in this scattering.

In Sec. II we review some of the experimental considerations. The elastic CC formalism is only briefly outlined in Sec. III since it has already been described elsewhere $[13,15,16]$. Theoretical results are presented and compared with the experimental ones in Sec. IV; this is followed by a discussion justifying our different interpretations and conclusions.

\section{EXPERIMENTAL CONSIDERATIONS}

The experiments we want to analyze have been described in detail in Refs. [5,6]. Experimental results have been obtained from a supersonic beam expansion of $\mathrm{He}$ atoms at different stagnation temperatures $T_{0}=300,50$, and $8.7 \mathrm{~K}$ corresponding to incidence wave vectors $k$ of 112,46 , and $18 \mathrm{~nm}^{-1}$, respectively. In order to maintain a high atomic flux and narrow velocity distribution of the incident beam and avoid cluster formation, different stagnation pressures $P_{0}=31,26$, and 0.5 bar have been used. In the cryogenic free jet expansion of incident particles, the incident kinetic energy is given by $E_{i}=(5 / 2) k_{B} T_{0}$ ( $k_{B}$ is Boltzmann's constant) [19]. Four types of surfaces have been considered in these experiments [6]: (i) a glass slide which is a simple standard microscope slide (ISO Norm 8037/I), $1 \mathrm{~mm}$ thick and with a surface area of $76 \times 26 \mathrm{~mm}^{2}$; (ii) the commercial GaAs wafer which is cut along the (100) direction and is $50 \mathrm{~mm}$ in diameter; (iii) a flat chromium surface of $100 \times 30 \mathrm{~mm}^{2}$ area used for comparison with the grating surface; and (iv) a 20- $\mu \mathrm{m}$-period chromium grating previously used in Ref. [5] (a 56-mm-long microstructured array of 110-nm-thick, 10$\mu \mathrm{m}$-wide, and 5-mm-long parallel chromium strips on a flat quartz substrate). Surfaces are expected to be oxidized or oxygen covered but, in spite of this contamination, at grazing angles intense specular reflection peaks are still observable. Since no information about the roughness of the surfaces employed in the experiments is provided, they are going to be assumed flat (except for type iv) and well described by one dimension in the $x$ direction. The incident angle $\theta_{i}$ is usually varied below $0.1 \mathrm{mrad}$ and up to $25 \mathrm{mrad}$ and measured with respect to the surface plane.

Angular distributions or diffraction patterns of in-saggitalplane $(x, z$-plane) scattering are recorded by rotating the detector and measuring the He signal at each angle. The diffraction angles $\theta_{n}$ are given by the conservation of the momentum or Bragg's law:

$$
\cos \theta_{i}-\cos \theta_{n}=\frac{n \lambda}{d}=\frac{2 \pi n}{d k},
$$

where $\lambda$ is the de Broglie wavelength of the incident particle and the diffraction order is given by $n$. Negative diffraction orders correspond to diffraction angles close to the surface grating, that is, energy in the perpendicular direction is transferred to the parallel direction. The specular reflection and nonspecular diffraction probabilities are obtained from the integrated intensity of the reflected peak normalized to the peak area of the incident beam and the total reflection probability is the sum of all of them. Final results are plotted as a function of the corresponding perpendicular wave vector, that is, along the $z$ direction:

$$
k_{\text {perp }} \simeq \frac{\sqrt{5 m k_{B} T_{0}}}{\hbar} \sin \theta_{i}
$$

with $m$ being the He-atomic mass.

The main conclusions of the authors of this experimental paper are that at low $k_{\text {perp }}$ values the reflection probability is dominated by quantum threshold reflection, which is manifested by a steep linear decrease of the reflection probability with increasing $k_{\text {perp }}$. At larger $k_{\text {perp }}$, the corresponding results start to fan out and are rationalized in terms of classical reflection from the inner region of the repulsive interaction potential. The dividing line between both regimes is claimed to be around $k_{\text {perp }}=0.3 \mathrm{~nm}^{-1}$ and independent of the type of surface studied. Only the fanning out effect is explained in terms of the surface roughness. 


\section{THEORY}

The theory employed here has been outlined elsewhere $[13,15]$ so we will emphasize only the key points:

(i) We assume an interaction model with very few free parameters.

(ii) The $\mathrm{CC}$ equations are solved numerically. The diffraction probabilities to be compared with the experimental ones are obtained after a fitting procedure of the fundamental potential parameters.

(iii) The final results are verified to be independent of the absorbing boundary conditions used. This assures that the internal turning point plays no role in the quantum reflection phenomenon.

A two-dimensional model potential between the incoming particles and the surface is assumed and written as

$$
U(x, z)=V(z) h(x)
$$

where $V(z)$ describes the interaction along the coordinate $z$ perpendicular to the surface and $h(x)$ is the periodic surface along the horizontal coordinate $x$. For the $z$ direction, the combination of a Morse potential, $V_{M}(z)$, at short distances, and an attractive van der Waals-Casimir tail $V_{C}$, at large distances, has been shown to be a good description of this interaction:

$$
V(z)=\left\{\begin{array}{ll}
V_{M}(z)=D\left[e^{-2 \chi z}-2 e^{-\chi z}\right], & z<\bar{z} \\
V_{C}(z)=-\frac{C_{4}}{(l+z) z^{3}}, & z \geqslant \bar{z}
\end{array} .\right.
$$

Here $C_{4}=C_{3} l, C_{3}$ being the van der Waals coefficient and $l$ a characteristic length which determines the transition from the van der Waals $(z \ll l)$ to the Casimir $(z \gg l)$ regime. The matching point $\bar{z}$ is usually determined by imposing both the continuity condition for the interaction potential and its first derivative. The range of variation of the $C_{3}$ parameter is usually known and the stiffness parameter of the Morse potential $\chi$ is considered a fitting parameter; $D$ is determined from the matching point $\bar{z}$.

The periodic grating function $h(x)$ is described by the socalled unit impulse function and written as

$$
h(x)=\sum_{n=-\infty}^{+\infty} \prod\left(\frac{x-n d}{a}\right)
$$

where $a$ is the width of the strips and $d$ is the period with $a<$ $d$. The $\prod(y)$ function is the so-called unit impulse function: zero for $|y|>1 / 2,1$ for $|y|<1 / 2$, and $1 / 2$ for $|y|=1 / 2$. In terms of a Fourier series, $h(x)$ is expanded as

$$
h(x)=\sum_{n=-\infty}^{+\infty} c_{n} e^{i 2 \pi n x / d}
$$

with $c_{0}=a / d, \quad c_{-n}=c_{n}$ and $c_{n}=(a / d) \operatorname{sinc}(n a / d)$, and $\operatorname{sinc}(x)=\sin (\pi x) / \pi x$. When $d=2 a$ (as in the experimental grating of Ref. [5]), the terms beyond the sixth order are quite small. The periodic interaction potential can then be expressed as

$$
U(x, z)=\sum_{n=-\infty}^{+\infty} V_{n}(z) e^{i \frac{2 \pi n x}{d}}
$$

where the first term $(n=0)$ is the interaction potential $V_{0}(z)=$ $V(z)$ [see Eq. (4)] and the coupling terms $(n \neq 0)$ are given by

$$
V_{n}(z)=2 \operatorname{sinc}\left(n \frac{a}{d}\right) V(z),
$$

where $d$ and $a$ are the period and width of the strips.

As has been recently shown $[13,15]$, the elastic scattering of the incident particles with the surface is theoretically well described by the $\mathrm{CC}$ formalism. The corresponding $\mathrm{CC}$ differential equations are written as

$$
\left[\frac{\hbar^{2}}{2 m} \frac{d^{2}}{d z^{2}}+\frac{\hbar^{2}}{2 m} k_{n, z}^{2}-V_{0}(z)\right] \psi_{n}(z)=\sum_{n \neq n^{\prime}} V_{n-n^{\prime}}(z) \psi_{n^{\prime}}(z)
$$

with $\frac{\hbar^{2}}{2 m} k_{n, z}^{2}$ being the $z$ component of the kinetic energy of the scattered particles. The square $z$ component of the wave vector is given by

$$
k_{n, z}^{2}=k_{i}^{2}-\left(k_{i} \sin \theta_{i}+\frac{2 \pi n}{d}\right)^{2}
$$

with $\theta_{i}$ measured with respect to the normal to the surface. This theoretical angle is complementary to the experimental incident angle. Thus, when comparing with experimental results, theoretical positive $n$ diffraction orders correspond to experimental negative ones. The effective potentials labeled by $n, V_{0}(z)+\frac{\hbar^{2}}{2 m}\left(k_{i} \sin \theta_{i}+2 \pi n / d\right)^{2}$ in Eq. (9) represent diffraction channels. The asymptotic energies depend on $n$ and the incident energy and polar angle. As is known, open (closed) diffraction channels have a positive (negative) normal kinetic energy $\hbar^{2} k_{n, z}^{2} /(2 m)$. The coupling between channels $V_{n-n^{\prime}}(z)$ is given by Eq. (8) since $n-n^{\prime}$ is always an integer number. The diffraction intensities or reflection probabilities, obtained by solving the CC equations given by Eq. (9) with the usual boundary conditions [16], are expressed as

$$
I_{n}=\left|S_{n 0}\right|^{2}
$$

where $S_{n n^{\prime}}$ are the elements of the unitary scattering matrix. Their square absolute values give the diffraction intensity or probability for an incident wave at the specular channel $\left(n^{\prime}=0\right)$ and exiting by any of the open diffraction channels labeled by $n$.

As mentioned above, the interaction potential given by Eq. (4) displays classical turning points due to the repulsive part of the Morse potential. To distinguish between quantum threshold reflection and classical reflection from the inner repulsive part of the Morse potential, absorbing boundary conditions have to be imposed $[17,18]$ in the inner part. A Woods-Saxon (WS) potential is added to the imaginary part of the diffraction channel potentials:

$$
V_{\mathrm{WS}}=\frac{A}{1+e^{\alpha \chi\left(z-z_{i}\right)}},
$$

which is essentially zero in the physically relevant interaction region and turns on sufficiently rapidly but smoothly at the left edge of the numerical grid for the integration to absorb the flux. The fitting parameters of this WS potential are $A$ and $\alpha$. The resulting scattering matrix $\bar{S}$ is then no longer unitary. The diffraction intensities are given by $\bar{I}_{n}=\left|\bar{S}_{n 0}\right|^{2}$ and the total 


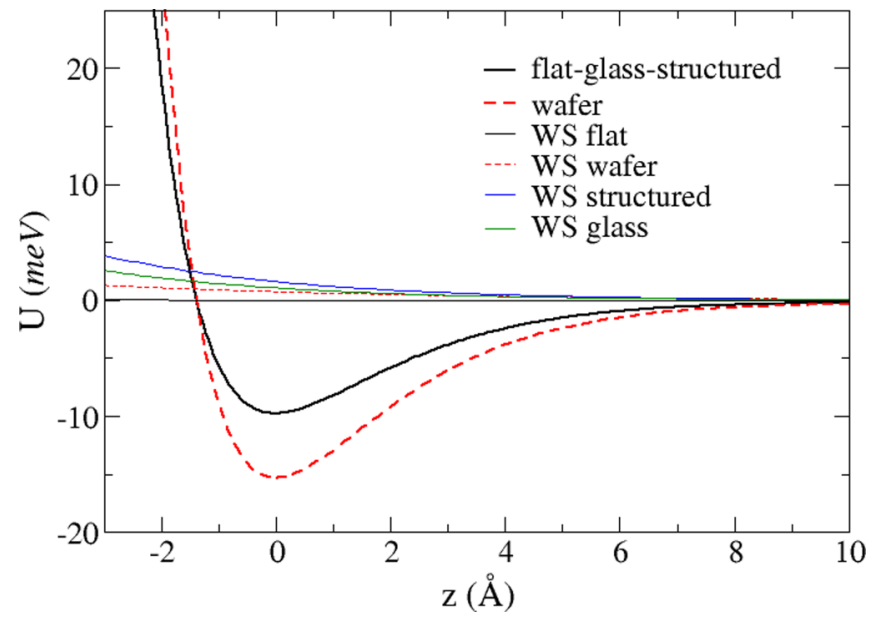

FIG. 1. The interaction potentials for the glass slide, flat, and structured $\mathrm{Cr}$ surfaces (solid curve) and wafer (dashed curve) are plotted. The WS potentials are also plotted for all the surfaces used in each case (gray curves).

quantum reflection probability is calculated as

$$
P^{\mathrm{QR}}=\sum_{n}\left|\bar{S}_{n 0}\right|^{2}<1
$$

for each initial condition. Due to the absorbing potential the theoretical diffraction efficiencies are defined as the ratio of the diffraction intensity $\bar{I}_{n}$ to the total quantum reflection probability $P^{\mathrm{QR}}$ rather than to the total incident flux in order to compare to the experimental results. In Fig. 1, the potentials for the glass slide, flat, and structured $\mathrm{Cr}$ surfaces are plotted together with the WS potentials used in each case. For flat surfaces, $h(x)=1$ and no diffraction channels are present; only specular reflection is present. The WS potential is then added to the specular channel.

\section{RESULTS AND DISCUSSION}

The parameters used in the elastic CC calculations for the perpendicular potential are displayed in Table I. As previously noted, the only real fitting parameter is $\chi$ since the values of $C_{3}$ have been taken from Ref. [6] and only slightly modified. The values obtained from fitting to the experimental results when solving numerically the one-dimensional Schrödinger equation only for the attractive potential were 3.5, 5.5, 3.5, $3.5 \times 10^{-50} \mathrm{~J} \mathrm{~m}^{3}$ for the glass slide, wafer, flat, and structured

TABLE I. Parameters of the interaction potential $V(z)$ for the four surfaces. The stiffness parameter of the Morse potential, $\chi$, is a free parameter fitted to reproduce the corresponding experimental results and $D$ is the well depth. The characteristic lengths $l$ and parameters $C_{3}$ are based on values reported in previous works [6].

\begin{tabular}{lcccc}
\hline \hline Parameters & Glass slide & GaAs wafer & Flat Cr & Structured Cr \\
\hline$\chi\left(\AA^{-1}\right)$ & 0.5 & 0.5 & 0.5 & 0.5 \\
$D(\mathrm{meV})$ & 9.8 & 15.3 & 9.8 & 9.8 \\
$l(\AA)$ & 93 & 93 & 93 & 93 \\
$C_{3}\left(10^{-50} \mathrm{~J} \mathrm{~m}^{3}\right)$ & 3.5 & 5.5 & 3.5 & 3.5 \\
\hline \hline
\end{tabular}

$\mathrm{Cr}$, respectively. The well depth is related to $\chi$ according to our procedure to evaluate the matching point $\bar{z}$. A characteristic length $l$ of $9.3 \mathrm{~nm}$ for He for the transition from the Casimir to the van der Waals regimes in the long-range attractive potential is a well-accepted value in the literature [6]. For the numerical integration, the range of distances in the vertical $z$ axis was taken to be -13 to $2000 \AA$. In this way the wave function was forced to vanish at $z=-13 \AA(z=0$ corresponds to the location of the minimum of the attractive Morse potential).

The parameters of the WS potential were varied with the incident wave vector. The detailed procedure for using these complex absorbing boundary conditions for the diffraction channels has been described elsewhere $[13,15]$. Due to the fact that no information is available on the structure of the experimental surfaces used, we have assumed that they are flat, except for the structured Cr surface. Thus, $h(x)=1$ is chosen for the glass slide, GaAs wafer, and flat $\mathrm{Cr}$ surfaces leading to only specular reflection or reflectivity. The characteristics of the grating $\mathrm{Cr}$ surface are the same as reported in Ref. [5] with $h(x)$ given by Eq. (6).

A measure of the quality of the reflection probability fits for each surface can be given by the square root of the relative deviation $\sigma$, which is defined as

$$
\sigma=\sqrt{\frac{1}{N(N-1)} \sum_{j=1}^{N}\left|\frac{P_{j}^{\exp }-P_{j}^{\text {theo }}}{P_{j}^{\exp }}\right|^{2}} .
$$

Here, $N$ is the total number of initial conditions given by the $k_{\text {perp }}$ points for each surface and $j=1, \ldots, N$. The smaller the sigma coefficient, the better the quality of the fit.

Reflection probabilities for He atoms scattering from a glass slide at three different source temperatures of $8.7 \mathrm{~K}$ (black labels), $50 \mathrm{~K}$ (red labels, light gray), and $300 \mathrm{~K}$ (blue labels, dark gray) are plotted in Fig. 2 as a function of $k_{\text {perp }}$ in $\mathrm{nm}^{-1}$ (top panel) and versus the incident angle in rad (bottom panel). Points are the experimental results and solid curves are our elastic $\mathrm{CC}$ results. The overall agreement is fairly good. The universal linear behavior of the quantum reflection probability in the top panel is observed for $k_{\text {perp }}$ values below $0.2 \mathrm{~nm}^{-1}$, depending on the stagnation temperature. After this small region of $k_{\text {perp }}$, one finds that the value of $k_{\text {perp }}$ causes this probability to fan out. The same is observed in the bottom panel where the incident angle also contributes to this effect. The quantum threshold reflection clearly decreases with $k$ and $\theta_{i}$. The $\sigma$ coefficient is 0.063 for $8.7 \mathrm{~K}, 0.143$ for $50 \mathrm{~K}$, and 0.114 for $300 \mathrm{~K}$.

Whereas the same observations are extracted from analyzing the experimental and theoretical results, our interpretation is different. We attribute our results to only quantum threshold reflection, not to classical reflection from the inner region of the potential even at higher $k_{\text {perp }}$. Our theoretical calculations are preventing classical reflection due to the use of the complex absorbing boundary conditions. The classical turning points of the perpendicular potential are not reached. The universal linear behavior is gradually lost with increasing $k_{\text {perp }}$. The transition from quantum to classical reflection cannot be observed by varying the incident wave vector. Moreover, from our calculations, it becomes clear that the fanning out 


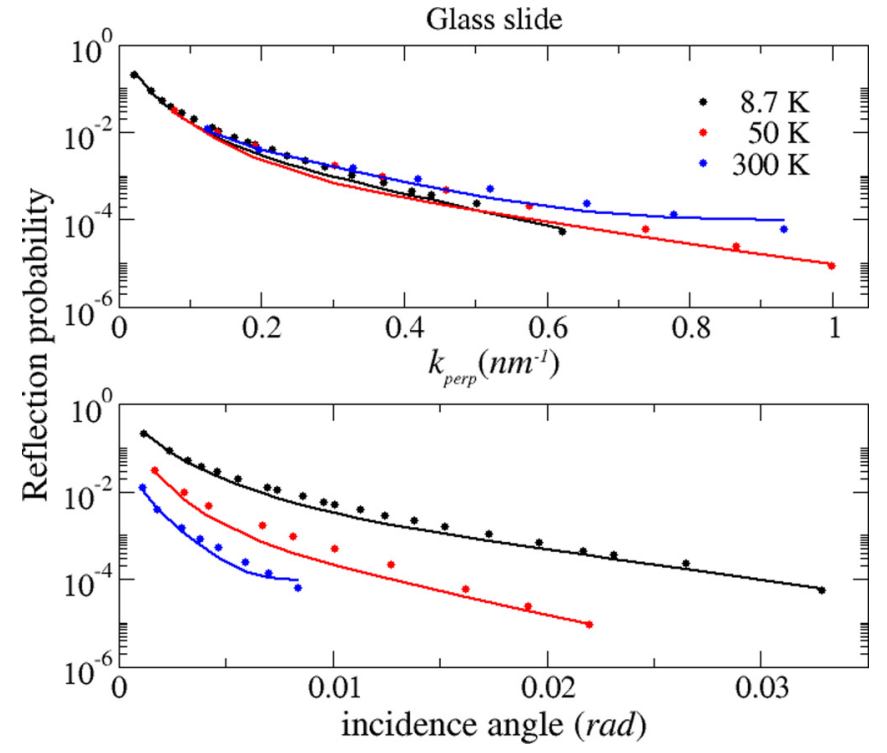

FIG. 2. Reflection probabilities for He atoms scattering from a glass slide at three different source temperatures $8.7 \mathrm{~K}$ (black labels), $50 \mathrm{~K}$ (red labels, light gray), and $300 \mathrm{~K}$ (blue labels, dark gray). In the top panel, probabilities are plotted as a function of $k_{\text {perp }}$ in $\mathrm{nm}^{-1}$ whereas in the bottom panel they are plotted vs the incident angle in rad. Points are the experimental results and solid lines show the results of the present elastic CC computations.

effect observed for this surface should not be attributed to the surface roughness as mentioned in Ref. [6] since we used a flat surface.

The same arguments and conclusions are valid also for the GaAs wafer surface, the results of which are plotted in Fig. 3. The degree of this fanning out is the smallest for the wafer as compared to the three other surfaces studied. The hierarchy of surface roughness determined by qualitative atomic force microscope measurement [6] indicates that the root-mean-square surface roughness is smallest for the chromium surface. In

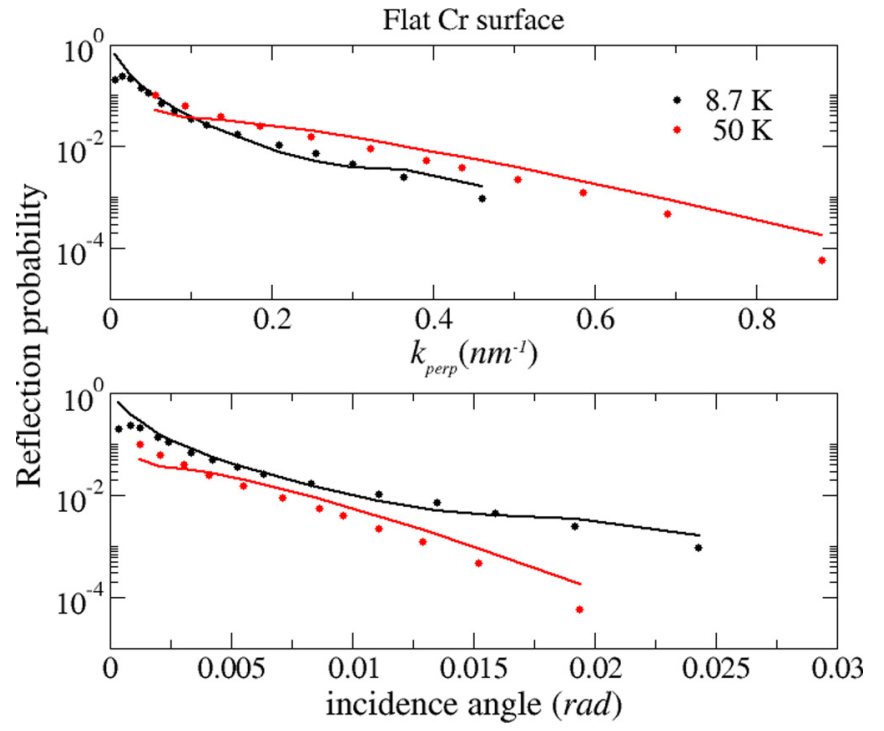

FIG. 4. The same as in Fig. 2 but for a flat Cr surface.

particular, the glass slide is larger than for the wafer. It is thus suggestive that the extent of surface roughness affects the extent of fanning out at higher $k_{\text {perp }}$. However, from our computations we conclude that this roughness has at most a minor effect on the quantum threshold reflection. The $\sigma$ coefficient is 0.044 for $8.7 \mathrm{~K}, 0.049$ for $50 \mathrm{~K}$, and 0.215 for $300 \mathrm{~K}$.

The next two surfaces are interesting to study to see what is the real effect of the roughness. In Figs. 4 and 5, we present the quantum reflection probabilities for a flat and structured chromium surface, respectively. The linear behavior seems to take place for perpendicular wave vectors less than $0.2 \mathrm{~nm}^{-1}$ in both cases. The fanning out effect is also more pronounced in the structured surface. However, the conclusions are the same as previously mentioned for the other two surfaces. The $\sigma$ coefficient is 0.09 for $8.7 \mathrm{~K}$ and 0.24 for $50 \mathrm{~K}$ for the flat

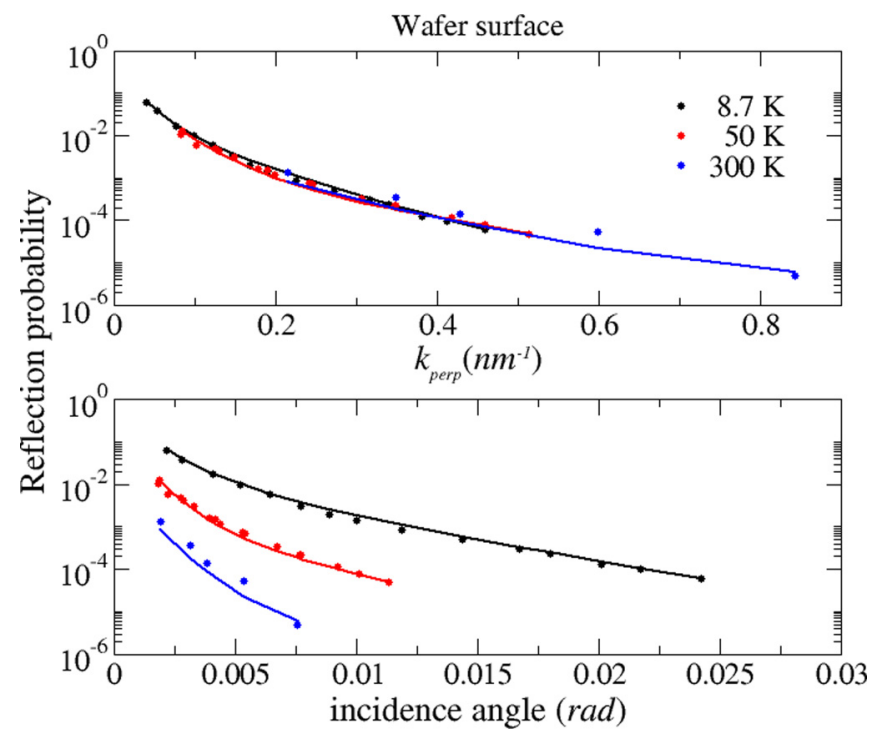

FIG. 3. The same as in Fig. 2 but for the GaAs wafer.

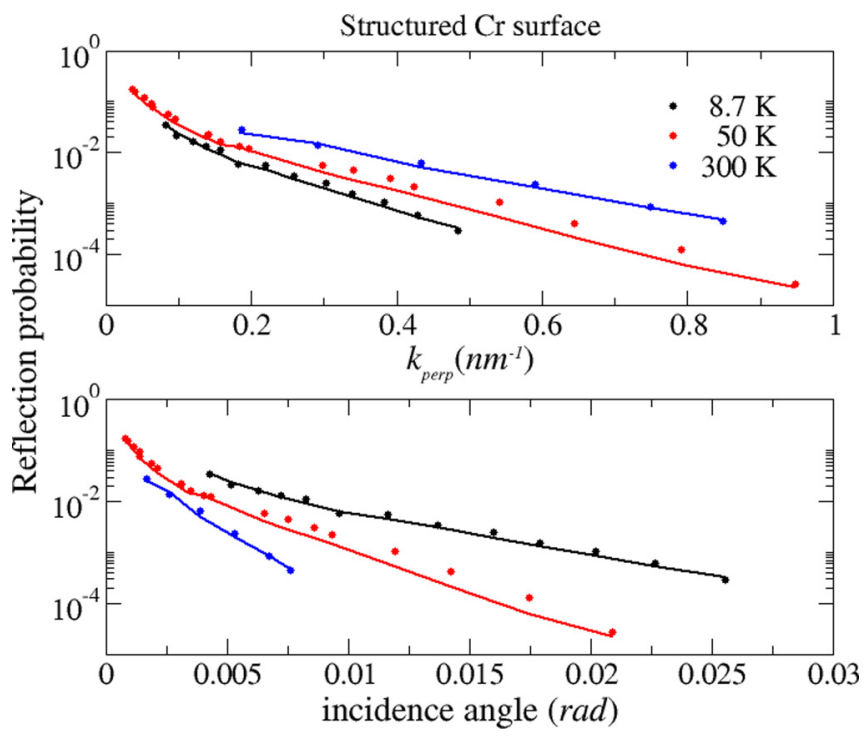

FIG. 5. The same as in Fig. 2 but for the structured Cr surface. 
surface, whereas for the structured surface we have 0.04 for $8.7 \mathrm{~K}, 0.063$ for $50 \mathrm{~K}$, and 0.058 for $300 \mathrm{~K}$.

\section{CONCLUDING REMARKS}

In this paper, we presented a theoretical study, based on the close-coupled equations method, of the scattering of He-atom beams at grazing angles and threshold conditions on four surfaces: a glass slide, a GaAs wafer, and a flat and structured chromium surface. The reasonable agreement between our results and the experimental ones indicates the reflection observed in the experiment should be attributed solely to quantum threshold reflection and not to classical reflection from the repulsive inner region of the potential. For all the surfaces studied we also observe the fanning out effect of the reflection probability with increasing incident energy but attribute this to the quantum threshold reflection rather than the classical reflection from the inner turning point. The universal linear dependence of the reflection probability on the perpendicular component of the incident wave vector is gradually lost. Finally, the decrease of the quantum threshold reflection probability with the incident wave vector and incident angle is found to be less pronounced for the wafer and much more for the structured $\mathrm{Cr}$ surface due to its roughness. The present computational result again indicates that the whole interaction potential is needed to correctly describe the quantum threshold reflection phenomenon, not only qualitatively but also quantitatively.

\section{ACKNOWLEDGMENTS}

We would like to thank W. Schöllkopf and B. S. Zhao for providing us with their experimental results. This work is supported by the Programa Nacional de Ciencias Básicas de Cuba under Grant No. P223LH001-108 (G.R.L. and J.R.S.); by the Ministerio de Ciencia, Innovación y Universidad (Spain) under Grant No. FIS2017-83473-C2-1-P (S.M.A.); and by grants from the Israeli Science Foundation and the Minerva Foundation, Munich (E.P.).
[1] J. E. Lennard-Jones and A. F. Devonshire, Proc. R. Soc. A 156, 6 (1936).

[2] W. Kohn, Surf. Sci. Lett. 01, 129 (1994).

[3] P. Senn, Am. J. Phys. 56, 916 (1988).

[4] H. Oberst, Y. Tashiro, K. Shimizu, and F. Shimizu, Phys. Rev. A 71, 052901 (2005).

[5] B. S. Zhao, S. A. Schulz, S. A. Meek, G. Meijer, and W. Schöllkopf, Phys. Rev. A 78, 010902(R) (2008).

[6] B. S. Zhao, H. Christian Schewe, G. Meijer, and W. Schöllkopf, Phys. Rev. Lett. 105, 133203 (2010).

[7] B. S. Zhao, G. Meijer, and W. Schöllkopf, Science 331, 892 (2011).

[8] B. S. Zhao, W. Zhang, and W. Schöllkopf, Sci. Adv. 2, e1500901 (2016).

[9] J. H. Lee, L. Y. Kim, Y.-T. Kim, C. Y. Lee, W. Schöllkopf, and B. S. Zhao, Phys. Rev. Lett. 122, 040401 (2019).
[10] H. Friedrich, G. Jacoby, and C. G. Meister, Phys. Rev. A 65, 032902 (2002)

[11] H. Friedrich and J. Trost, Phys. Rep. 397, 359 (2004).

[12] A. R. Barnea, B. A. Stickler, O. Cheshnovsky, K. Hornberger, and U. Even, Phys. Rev. A 95, 043639 (2017).

[13] S. Miret-Artés and E. Pollak, J. Phys. Chem. Lett. 8, 1009 (2017).

[14] J. Petersen, E. Pollak, and S. Miret-Artés, Phys. Rev. A 97, 042102 (2018).

[15] G. Rojas-Lorenzo, J. Rubayo-Soneira, S. Miret-Artés, and E. Pollak, Phys. Rev. A 98, 063604 (2018).

[16] A. S. Sanz and S. Miret-Artés, Phys. Rep. 451, 37 (2017).

[17] T. Seideman and W. H. Miller, J. Chem. Phys. 96, 4412 (1992).

[18] J. G. Muga, J. P. Palao, B. Navarro, and I. L. Egusquiza, Phys. Rep. 395, 357 (2004)

[19] O. Kornilov and J. P. Toennies, Europhysics News 38, 22 (2007). 